\title{
Functional dissection of TADs reveals non-essential and instructive roles in regulating gene expression
}

Alexandra Despang ${ }^{1,2,3}$, Robert Schöpflin ${ }^{1,2,3}$, Martin Franke ${ }^{1, \S}$, Salaheddine Ali ${ }^{1,2,3}$, Ivana Jerkovic ${ }^{1,2}$, Christina Paliou ${ }^{1,2}$, Wing-Lee Chan ${ }^{2}$, Bernd Timmermann ${ }^{4}$, Lars Wittler ${ }^{5}$, Martin Vingron $^{6}$, Stefan Mundlos ${ }^{1,2,3}{ }^{*}$, Daniel M. Ibrahim ${ }^{1,2,3}$ *

${ }^{1}$ RG Development \& Disease, Max Planck Institute for Molecular Genetics, 14195 Berlin, Germany

${ }^{2}$ Institute for Medical and Human Genetics, Charité Universitätsmedizin Berlin, 13353 Berlin, Germany

${ }^{3}$ Berlin-Brandenburg Center for Regenerative Therapies (BCRT), Charité Universitätsmedizin Berlin, 13353 Berlin, Germany

${ }^{4}$ Sequencing Core Facility, Max Planck Institute for Molecular Genetics, 14195 Berlin, Germany

${ }^{5}$ Department of Developmental Genetics, Max Planck Institute for Molecular Genetics, 14195 Berlin, Germany

${ }^{6}$ Department of Computational Molecular Biology, Max Planck Institute for Molecular Genetics, 14195 Berlin, Germany

$\S$ current address: Centro Andaluz de Biología del Desarrollo (CABD), Consejo Superior de Investigaciones Científicas/Universidad Pablo de Olavide/Junta de Andalucía, Sevilla, Spain

*corresponding authors: ibrahim@molgen.mpg.de, mundlos@molgen.mpg.de 


\section{Abstract}

The genome is organized in megabase-sized three-dimensional units, called Topologically Associated Domains (TADs), that are separated by boundaries. TADs bring distant cisregulatory elements into proximity, a process dependent on the cooperative action of cohesin and the DNA binding factor CTCF. Surprisingly, genome-wide depletion of CTCF has little effect on transcription, yet structural variations affecting TADs have been shown to cause gene misexpression and congenital disease. Here, we investigate TAD function in vivo in mice by systematically editing components of TAD organization at the Sox9/Kcnj locus. We find that TADs are formed by a redundant system of CTCF sites requiring the removal of all major sites within the TAD and at the boundary for two neighboring TADs to fuse. TAD fusion resulted in leakage of regulatory activity from the Sox9 to the Kcnj TAD, but no major changes in gene expression. This indicates that TAD structures provide robustness and precision, but are not essential for developmental gene regulation. Gene misexpression and resulting disease phenotypes, however, were attained by re-directing regulatory activity through inversions and/or the re-positioning of boundaries. Thus, efficient re-wiring of enhancer promoter interaction and aberrant disease causing gene activation is not induced by a mere loss of insulation but requires the re-direction of contacts. 


\section{Introduction}

Chromosomes are organized in a specific three dimensional (3D) structure in the nuclear space, a phenomenon that is directly linked to gene regulation reviewed $\mathrm{in}^{1} ;{ }^{2,3}$. On a gene locus-level, this organization is characterized by regions of high interaction called Topologically Associating Domains (TADs) that are separated from each other by so-called boundaries ${ }^{4,5}$. TAD bring distant cis-regulatory elements such as promoters and enhancers into proximity whereas boundaries are thought to act as insulators to preclude inappropriate enhancer-promoter interactions with neighboring genes or regulatory elements. This concept provides a basic framework for long range gene regulation ${ }^{6}$ and also has important implications for the interpretation of genomic rearrangements (structural variations) reviewed in ${ }^{7}$.

One key component for TAD and boundary formation is the zinc finger transcription factor CTCF, which acts in concert with the multi-subunit protein complex Cohesin ${ }^{8}$. In the currently prevailing model, TAD formation is the result of a loop extrusion process in which cohesin molecules extrude a chromatin loop and thereby bring distant DNA fragments into spatial proximity ${ }^{9,10}$. In this model CTCF binding sites act as a barrier for the extrusion machinery in an orientation-dependent manner. This view is supported by the finding that a large fraction of TAD boundaries harbor clusters of CTCF binding sites that are characteristically positioned in divergent orientation ${ }^{3,9}$.

The importance of the CTCF/cohesin machinery for higher order chromatin architecture has further been corroborated by experimental approaches that allow for the temporary genome-wide depletion of CTCF or various subunits of the cohesin complex, circumventing their absolute requirement for cell survival ${ }^{8,11-14}$. Cells in which CTCF or cohesin is depleted loose most of their TAD structures. In spite of this dramatic loss in 3D genome organization, however, only modest effects on gene expression were observed ${ }^{8,11,14}$. Less than half of the regulated genes exhibit elevated expression, suggesting only spurious gains in enhancerpromoter interactions in the absence of TADs and boundaries. These results are in contrast to previous findings in which the rearrangement of TADs and their boundaries were shown to have dramatic effects on gene regulation resulting in congenital disease or cancer ${ }^{15-17}$. The basis for this apparent discrepancy remains unclear, raising the question about the functional importance of TADs for gene regulation and the proposed molecular pathology of SVs.

Here, we dissect the role of CTCF and TAD architecture for gene regulation in a developmental in vivo setting in mice. We created a series of genome-engineered mice with targeted mutations at the Sox9/Kcnj locus and analyzed their effect on 3D chromatin architecture, gene regulation, and the phenotype. The Kcnj and Sox9 TADs are separated by 
a strong boundary, but a fusion of the TADs, as indicated by HiC, was only achieved after removal of all major CTCF sites at the boundary and within the TAD. TAD fusion, however, was not accompanied by major gene regulatory effects, suggesting that long-range gene regulation does not exclusively rely on intact TAD structures. In contrast, inversions and the insertion of boundary elements were able to re-direct regulatory activity inducing enhancerpromoter rewiring, gene misexpression and developmental phenotypes. Thus, TADs, and in particular CTCF sites are not essential for correct developmental gene expression but they can induce gene misexpression when re-directed.

\section{Results}

\section{Two TADs define the regulatory landscape at the Sox 9 and Kcnj2 locus}

Sox9 and Kcnj2 are two adjacent genes with distinct expression patterns in the developing limb bud that are separated by a 1.7 Mb gene desert (Fig. 1). In E12.5 limb buds Sox9 is expressed in the cartilage anlagen of the developing limbs, whereas Kcnj2 is only weakly expressed in the distal zeugopod (Fig. 1b). Capture HiC (cHiC) from mouse limb buds shows that the locus is divided in two TADs, one harboring Sox 9 and the other Kcnj2 and Kcnj16 ${ }^{15}$. The TAD boundary is characterized by two pairs of divergent CTCF binding sites with divergent orientation, showing strong loop formation with their neighboring boundaries (Fig. 1). Within the Sox9-TAD a nested substructure with various loops is linked to at least four additional CTCF binding sites (C1 to $\mathrm{C} 4$ ). To profile the regulatory landscape of the locus in more detail, we performed ATAC-seq and H3K27ac ChIP-seq from E12.5 limb buds and identified many putative enhancer regions carrying active enhancer marks (Fig. 1a). To capture the cis-regulatory activity of the locus we investigated regulatory sensors ( $\beta$ globin minimal promoter with LacZ reporter gene), integrated at various positions within the Kcnj and Sox9 TADs (Fig. 1b). LacZ staining of E12.5 embryos revealed that all sensors within the Sox9 TAD recapitulated the Sox9 expression pattern, whereas sensors integrated within the Kcnj TAD reflected the endogenous expression pattern of Kcnj2. This shows that the 3D genome organization in the two TADs corresponds with the regulatory domains of the Kcnj2 and Sox9 genes.

\section{Boundaries and internal CTCF sites act cooperatively to form TADs}

To investigate the role of CTCF in maintaining TAD structure at this locus, we produced a series of alleles in which the 4 CTCF sites at the TAD boundary were deleted, followed by a consecutive deletion of five further sites within the TADs. CHiC was performed from E12.5 limb buds to visualize the effects on TAD architecture and to quantify the contacts within 
the TADs (intra-TAD) and between the TADs (inter-TAD). We also produced virtual 4C interaction profiles from the $\mathrm{CHiC}$ data to assess contact changes of Sox9 and Kcnj2 in these alleles, (see Methods).

Deletion of the 4 CTCF sites that constitute the $K c n j / S o x 9-T A D$ boundary $(\triangle B o r)$ resulted in a moderate increase of contacts between TADs, but the two TADs remained largely separate (Fig. 2a, b) ${ }^{15}$. To test whether the intra-TAD CTCF sites contribute to TAD formation we sequentially deleted, in addition to the boundary, one ( $\triangle B$ orC1), two, and all 4 ( $\triangle B$ BorC1-4) of the major CTCF sites within the Kcnj/Sox9-TAD. Deletion of the C1 CTCF site together with the boundary deletion ( $\triangle B$ Bor $C 1$ ) led to a marked increase in contacts between the Sox9- and Kcnj-TADs, which became even more pronounced by the additional deletion of the C2 CTCF site ( $\triangle B$ BorC1-2) (Fig. S1). Deletion of all 4 CTCF sites ( $\triangle B$ BorC1-4) led to a further increase in inter-TAD contacts and a near complete fusion between the Sox9 and Kcnj-TADs (Fig. 2c, Fig. 3a). Moreover, while the internal TAD structure and loops disappeared, new interactions between the Sox9 and Kcnj2 promoters, as well as between the outer TAD boundaries, emerged. Further deletion of the remaining single major CTCF site in the Kcnj-TAD ( $\triangle C T C F)$ abolished all major CTCF binding sites between the Sox9 and Kcnj2 promoters. $\mathrm{cHi}-\mathrm{C}$ from these animals showed a further increase of inter-TAD contacts between the former Sox 9 and Kcnj-TADs (Fig. 2d, Fig. 3a). Taken together, our data show that the TAD boundary deletion alone does not result in TAD fusion. Instead, TAD formation and integrity is established by the TAD boundary in combination with the CTCF-mediated TAD substructure.

\section{Loss of TAD insulation has minor effects on developmental gene regulation}

We next investigated how the gradual fusion of TADs might affect the expression of Kcnj2 and Sox 9 and the phenotype. We previously demonstrated that misexpression of $K c n j 2$ in a Sox9-like pattern results in a malformation of the terminal phalanges (in humans called Cooks syndrome, OMIM 106995) ${ }^{15}$. Heterozygous loss of Sox9, on the other hand, leads to a lethal skeletal phenotype characterized by bowing of long bones, cleft palate, and rib abnormalities in heterozygous animals (in humans called campomelic dysplasia, OMIM 114290) and homozygous mutants do not form cartilage at all ${ }^{18}$. In addition, Sox 9 is an essential factor for testis development downstream of SRY and its inactivation results in male-to-female sex reversal ${ }^{19}$. To monitor gene expression changes in embryos we used qPCR and whole-mount in situ hybridization (WISH). Phenotypes associated with loss of Sox 9 and/or gain of Kcnj2 were assessed in mice by visual inspection (palate, claws), skeletal preparations, $\mu \mathrm{CT}$ (digits) and by testing fertility through breeding.

Despite the observed fusion of TADs, we did not observe any dramatic changes in gene expression. While the changes in Sox9 gene expression were not significant in the boundary 
deletion or the $\triangle B$ orC1-4 mutants, we detected a $\sim 10-15 \%$ reduction of Sox9 in the $\triangle C T C F$ animals (Fig. 3b, Fig. S2a). Kcnj2, which is only marginally expressed at this timepoint, increased slightly to approx. 2-fold in all alleles. To detect if there were changes in the patterns of expression in developing limbs, we performed WISH in E12.5 embryos. In all lines, Sox9 expression was indistinguishable from wildtype embryos. Also, Kcnj2 stayed unchanged and no Sox9-like misexpression was detected in $\triangle B$ or or $\triangle B o r C 1-2$ animals (Fig. 3a, Fig. S2b). However, upon deletion of four or more CTCF sites in addition to the TAD boundary faint Kcnj2 expression in the digit anlagen was detected ( $\triangle B$ BorC1-4 and $\triangle C T C F$, Fig. 3c). Importantly, despite the fusion of TAD structures, all mutant animals were viable, bred to Mendelian ratios, and had no detectable phenotype. In particular, no abnormality of the digits/claws was observed (Fig. S2c).

Thus, in spite of the observed TAD fusion, Sox 9 and Kcnj2 expression remained largely unchanged with no phenotypic effect. These results indicated that enhancers were able to efficiently contact their target gene even without CTCF loops. Removal of all CTCF sites, however, led to a spill over of activity from the Sox9 TAD to the Kcnj TAD indicating that TAD genome organization provides a certain degree of robustness and precision to gene expression at this locus.

\section{Re-organization of 3D chromatin contacts by TAD boundaries and TAD substructure orientation}

TAD fusions induced by large structural variations have been reported to cause gene misexpression and disease ${ }^{16}$, yet the fusion of TADs induced by CTCF site deletion occurred without major gene misexpression. To investigate this discrepancy further, we produced four different types of inversions/insertions: 1) an inversion of the Sox9 regulatory domain including the TAD boundary $(\operatorname{InvC}), 2$ ) an inversion of the Sox9 regulatory domain without the boundary (Inv-Intra), 3) an insertion of the boundary alone without inverting the regulatory domain (Bor-KnockIn), and, finally, 4) an inversion of the Sox9 regulatroy domain with the boundary removed (InvCABor) (summarized in Fig. S3). This combination of alleles allowed us to dissect the role of TAD boundaries and substructure orientation in TAD formation and for structural variations.

$\mathrm{CHiC}$ from the inversion of the centromeric $1.1 \mathrm{Mb}$ of the Sox9-TAD including the TAD boundary $(\operatorname{InvC})$ (E12.5 limb buds) showed a fusion of the inverted part of the Sox9-TAD with the Kcnj-TAD and a separation of the Sox9 gene and its remaining TAD from the inverted part (Fig. 4a, b). cHiC and virtual 4C showed that Kcnj2 was now able to contact the C1-C4 CTCF sites in a similar fashion as Sox9 in the wildtype situation (Fig. 4a, b). 
To investigate the effect of the inverted TAD substructure vs. the repositioned boundary we produced a slightly smaller inversion not including the boundary (Inv-Intra). cHiC of this intra-TAD inversion showed that the overall extent of interactions did not change. However, the re-direction of CGTCF sites resulted in an altered pattern of loop formation (Fig. 4c). The contacts between intra-TAD CTCF sites and the TAD boundary became stronger, while those with the Sox9 promoter became weaker, as if the entire region had shifted its interaction towards the TAD boundary (Fig. S4).

To test the effect of the TAD boundary alone we used the boundary deletion background to insert a $6.3 \mathrm{~kb}$ construct carrying the four boundary CTCF sites (schematic shown in Fig. S5d) $125 \mathrm{~kb}$ upstream of Sox9 (Bor-KnockIn). CHiC of this allele showed that the repositioned TAD boundary split the Sox9-TAD into two domains. Similar to the TAD-spanning InvC inversion, the telomeric region containing the Sox9 gene was now separated from the centromeric region (Fig. 4d). However, in contrast to the InvC inversion, the centromeric Sox9-TAD did not fuse with the Kcnj2-TAD but remained an isolated domain extending from the C1-CTCF site to the inserted boundary. Thus, the boundary was fully functional even at a different position separating the Sox9 TAD into two domains.

Finally, we wanted to test the effect of TAD substructure orientation without the influence of a nearby boundary. Deletion of the TAD boundary in the InvC inversion (InvCABor) resulted in a loss of insulation and again of Sox9 contacts with its centromeric TAD. At the same time the contacts between the inverted part of the Sox9-TAD and Kcnj2 were still present (Fig. 4e). Thus, this mutant resulted in a fusion of the entire Sox9 and Kcnj-TADs and both, Sox9 and Kcnj2, were contacting the inverted Sox 9 regulatory region.

To compare how TAD orientation and boundary position affect the contact frequency we quantified the contacts of the Sox 9 and Kcnj2 promoters with the inverted region (centromeric part of the Sox9-TAD) using virtual 4C (Fig. 4f). Analysis of the TAD-spanning inversion $(\operatorname{InvC})$ and the boundary knock-in (Bor-KnockIn) showed that the repositioned boundary caused a strong reduction of contacts of Sox 9 with its domain, a phenomenon that was not observed when only the TAD substructure was inverted (Inv-Intra and InvCABor). In contrast, inversion of the TAD substructure ( $\operatorname{lnvC}$ and $\operatorname{lnvC} \triangle B$ Bor) resulted in a gain of contacts of Kcnj2 with the centromeric Sox9-TAD provided that the two regions were not separated by a bondary.

\section{Re-direction of TAD structure results in distinct regulatory effects and phenotypes}

In contrast to the CTCF site deletions, the rearrangements produced pronounced regulatory and phenotypic effects. Animals carrying the $\operatorname{Inv} C$ allele, in which the inverted centromeric Sox9-TAD fused with the Kcnj-TAD, showed a clear loss-of-function of Sox9 and a gain-of- 
function of Kcnj2. Sox 9 expression decreased by $20 \%$ in heterozygous and $50 \%$ in homozygous animals (Fig. 5b, Fig. S5a). As a consequence, heterozygous animals displayed delayed ossification of the skeleton and homozygous animals showed perinatal lethality with all hallmarks of a Sox9 loss-of-function phenotype (i.e. bowing of long bones, delayed ossification, cleft palate)(Fig. S5c). Kcnj2 expression increased up to 5-fold in homozygous embryos (Fig. 5b, Fig. S5a). WISH in E12.5 embryos revealed a strong misexpression of Kcnj2 in a Sox9-like pattern in the digit anlagen (Fig. 5c). Heterozygous InvC animals showed malformed terminal phalanges with high penetrance, the phenotype associated with Kcnj2misexpression in a Sox9 like pattern. Homozygous animals had severely dysplastic digits preventing the development of this phenotype. Thus, the TAD-spanning inversion InvC reorganized the TADs at the locus and re-directed regulatory activity from Sox9 to Kcnj2.

In contrast, animals with the slightly smaller Inv-Intra inversion had no abnormal phenotype. Mice were viable and fertile suggesting no major misregulation of Sox9 or Kcnj2, which was confirmed by qPCR and WISH (Fig. 5b,c). These results indicate that the orientation of the internal TAD structure has no major effect as long as the boundary is intact.

Consistent with the $\mathrm{cHiC}$ pattern, the knock-in of the border (Bor-KnockIn) showed a Sox 9 loss-of-function, but no Kcnj2 gain-of-function. Sox9 levels were reduced by $40 \%$ in homozygous E13.5 limb buds (Fig. 5b) and Kcnj2 was upregulated 2-fold, similar to the CTCF-deletion alleles and WISH showed no Sox9-like Kcnj2 misexpression in E12.5 limb buds (Fig. 5a,c). Phenotypically, homozygous Bor-KnockIn animals died perinatally due to Sox9 related defects including cleft palate, short snout, shortened long bones, and delayed ossification (Fig. S5d). However, the skeletal phenotypes were less severe than those seen in the InvC animals. Importantly, in accordance with the regulatory effects of the boundary knock-in, the animals had normal phalanges (Fig. S5d).

Finally, we tested gene expression and phenotypes in the InvC $\Delta B$ or allele, in which the entire Kcnj- and Sox9-TADs are fused and the centromeric Sox9-TAD contacted both Sox9 and Kcnj2. Consistent with the re-established $\mathrm{cHiC}$ interactions, Sox9 expression was less severely reduced than in the InvC inversion (Fig. 5b, Fig. S5a) and the Sox9 expression pattern in E12.5 WISH was indistinguishable from wildtype Sox9 expression (Fig. 5c). On a phenotypic level, the InvC $\triangle B$ or allele rescued the Sox9 loss-of-function effects of the InvC inversion. Homozygous InvC $\triangle B$ or animals had no cleft palate and were viable. The only obvious Sox9 related phenotype of this allele was that homozygous males were infertile (Fig. 5c). Nonetheless, the Kcnj2 gain-of-function effects were still present. E13.5 Kcnj2 expression levels were 2.5 -fold higher than wildtype, but lower than in the InvC allele (Fig. 5b, Fig. S5a). WISH, however, showed a clear Sox9-like expression pattern in the limb buds. 
Consistent with this Kcnj2 misexpression, InvC $\Delta B$ Bor animals showed the abnormal terminal phalanx phenotype (Fig. S5b).

Thus, phenotype inducing alterations in gene expression can be caused by a change in directionality of the TAD substructure (including their CTCF sites) and/or the repositioning of boundary elements. Such re-directing of regulatory activity can result in either a gain of expression if enhancers are forced to act on a neighboring gene, or in a loss if a gene is disconnected its regulatory domain.

\section{Discussion}

Here we functionally dissect the role of TAD boundaries, intra-TAD CTCF sites and directionality of TAD substructures for TAD formation and gene regulation in a developmental in vivo model at the Kcnj-Sox9 locus. With its two large TADs and the distinct expression patterns of its corresponding genes as well as associated phenotypes, this region is ideally suited for this approach.

\section{TADs are formed by a redundant system of CTCF sites}

The deletion of the Kcnj-Sox9-TAD boundary did not have a major effect on the overall TAD configuration. To achieve effective TAD fusion, intra-TAD CTCF sites needed to be deleted in addition to the TAD boundary. Thus, we demonstrate a redundancy in spatial separation of TADs that originates from the combinatorial action of CTCF sites at the TAD boundary and within the TAD. A similar resilience of TAD structures was recently reported for TADs at the HoxD gene cluster, which itself acts as a strong TAD boundary. Here, only a $400 \mathrm{~kb}$ deletion encompassing the entire HoxD cluster and two flanking genes leads to fusion of the centromeric and telomeric HoxD-TADs ${ }^{20}$. Smaller deletions do not affect the spatial separation of the two adjacent TADs, supporting our finding that TADs are formed by redundant and buffered mechanisms. Similarly, at the EphA4 locus the presence of a TAD boundary in pathogenic deletions determines whether two TADs fuse, demonstrating that it functions as a potent insulator. The resulting TAD fusion at the EphA4 locus leads to misexpression of Pax3. This, however, is the result of a deletion that removes not only the boundary but, in addition, the majority of the Epha4 TAD and alters the entire 3D structure at the locus. In contrast, the serial deletions of CTCF-sites here leave the overall configuration of the locus intact but modify the barrier function between TADs. We thereby provide direct evidence that TADs are formed by a redundant system of CTCF sites at the TAD boundaries and within the TADs. 


\section{Insulation between TADs is not required for developmental gene regulation}

We addressed the role of CTCF at an individual locus in an in vivo developmental setting and avoid the genome wide effects associated with a loss of CTCF. Surprisingly, the gradual fusion of the two neighboring TADs was accompanied by only mild effects on gene regulation, indicating that the enhancers were able to find, contact, and regulate their cognate promoters. Surprisingly, this loss of TAD structures at the Sox9 locus also had no phenotypic consequences, indicating that there were no substantial effects on Sox9 or Kcnj2 regulation throughout development. These results are in agreement with the weak gene expression changes observed upon CTCF depletion ${ }^{8,14}$, suggesting that enhancer-promoter communication can function independent of TADs and CTCF-mediated genome architecture. Such a mechanism might be mediated by homotypic interaction of TFs that bind at distal enhancers and their cognate promoters and could promote transcriptional condensates as recently proposed ${ }^{21}$. In such a scenario, TADs would act as three-dimensional scaffolds that optimize such interaction hubs, without being essential to establish them.

Another reason for the mild effects on gene regulation is likely that the deletion of CTCF sites does not affect cohesin recruitment to the chromatin, which is independent of CTCF ${ }^{22}$. Thus, cohesin complexes at the Sox9 locus can still facilitate enhancer-promoter interaction. What does change, however, are the limits for the extrusion complexes that are normally set by CTCF. The consecutive removal of the boundary and intra-TAD CTCF sites leads to ever increasing contacts of Kcnj2 with the active Sox9 regulatory landscape. However, this only results in an incremental increase in Kcnj2 expression indicating that spurious contacts do not directly result in gene regulation.

However, it has to be pointed out that the loss of CTCF/insulation was accompanied a loss of Sox 9 and gain of Kcnj2 expression induced by a spread of regulatory activity from the Sox 9 to its neighboring TAD. Thus, TADs and their boundaries are probably not essential for developmental gene expression, but they confer precision and robustness to the system. At the locus investigated here, the relatively mild expression changes had no phenotypic effect. However, in other cases where precision and insulation is essential, such leaky expression might result in disease phenotypes.

Our findings support the idea that the spatial separation into TADs and enhancer-promoter interaction represent two independent layers of long range gene regulation. These layers stabilize each other but are not inherently linked. Furthermore, they contradict the generally accepted idea that enhancers are promiscuous ${ }^{6}$, i.e. can and will activate any promoter in their vicinity. The readiness to become activated by spurious enhancer contacts is likely to depend on various mechanisms including enhancer-promoter specificity ${ }^{23}$, histone 
modification, proximity to the target promoter, and openness of chromatin (Kraft et al. 2019).

\section{Boundaries and the orientation of substructures organize regulatory domains}

With our series of inversions and knock-in alleles, we were able to dissect the relevance of TAD substructure and TAD boundaries for altering gene expression. In the TAD-spanning InvC inversion the repositioned TAD boundary serves as a strong insulator separating Sox 9 from its regulators. At the same time, re-direction of the TAD substructure creates new loops with the Kcnj2 gene and fuses the Sox9 regulatory domain with the Kcnj2-TAD. This combination is apparently sufficient to connect the Sox9 enhancers to the Kcnj2 promoter, thereby overcoming their inherent affinity for the Sox 9 promoter. As a consequence, we observe a Sox 9 loss of function phenotype and a Kcnj2 gain of function. Thus, misexpression and disease can be induced by redirecting TAD substructures and enhancer activity but not by removing them. In this context, boundary and TAD substructure function together, but the substructure with its CTCF sites cannot override a boundary. However, its inversion is needed to achieve pathogenic misexpression.

Our results help to explain the apparent discrepancy between the modest effects of CTCF/cohesin depletion on transcription and the drastic effects of TAD reorganization in pathogenic structural variations. Based on our findings it is to be expected that, due to the high redundancy of CTCF sites in maintaining TAD structure, most SVs are likely to be tolerated. To result in aberrant gene activation, the rearrangement needs to actively reorganize 3D chromatin contacts and thereby connect a regulatory region to the new target gene. Such effects can be achieved by deleting boundaries together with adjacent divergent CTCF sites, or by re-directing regulatory activity through inversions or duplications. The repositioning of boundaries, on the other hand, can result in loss of expression induced by disconnecting a gene from its regulatory domain. Thus, SV induced misexpression is not caused by the simple removal of barriers or the effect single enhancer-promoter rewiring. Rather, it is the result of connecting larger regulatory structures with novel target genes through CTCF mediated loops. 


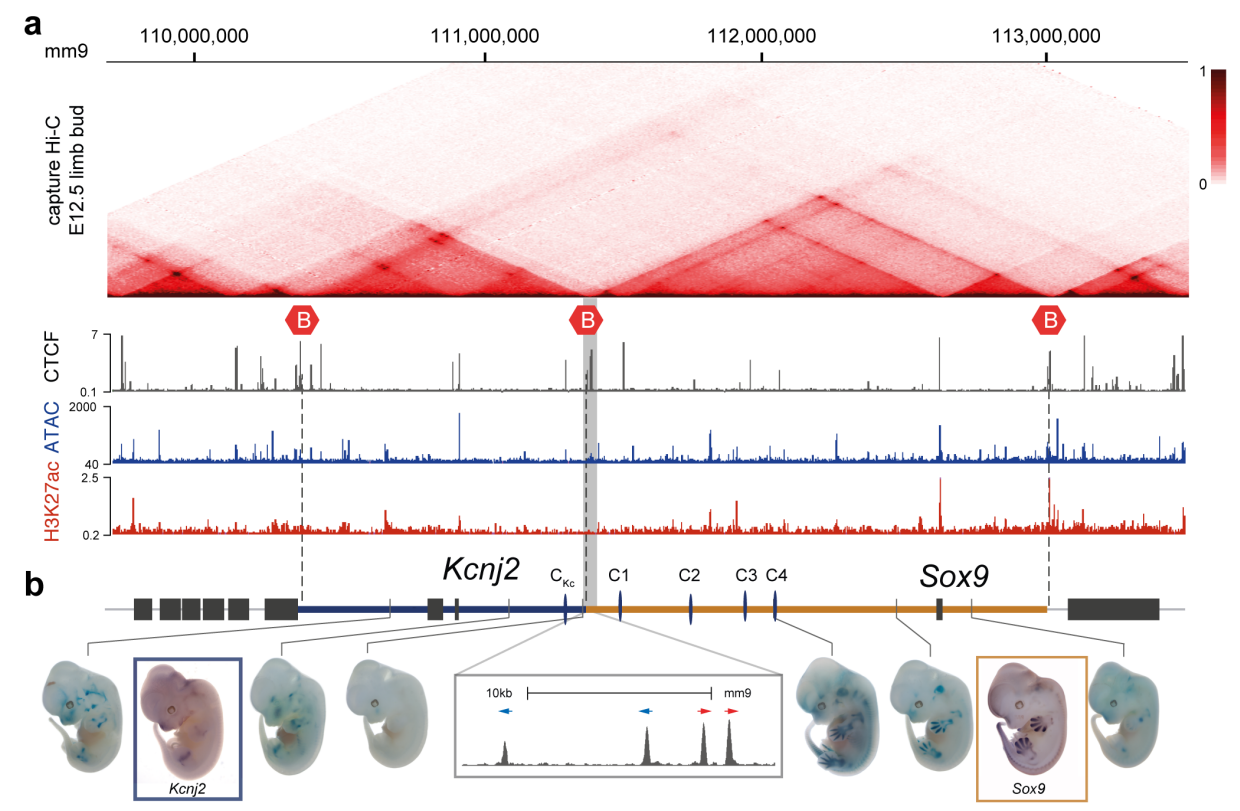

Figure 1: TAD configuration and regulatory activity at the Sox9-Locus.

(A) Capture HiC from E12.5 mouse limb buds with boundaries indicated by red hexagons. CTCF ChIP-seq, ATAC-seq and H3K27ac shown below. Note multiple CTCF sites at boundaries and within TADs as well as potential cis-regulatory elements indicated by ATAC-seq tracks and H3K27ac-ChIP-seq peaks. (B) Schematic of the locus. Genes are indicated by black bars, TADs of Kcnj2 (blue) and Sox9 (orange). Boundary region between Sox9 and Kcnj-TADs is highlighted in grey, magnification below shows the cluster of 4 divergent CTCF sites within a $15 \mathrm{~kb}$ region. Other major CTCF-binding sites are indicated and labeled as C1, C2, C3, C4 and $\mathrm{C}_{\mathrm{Kc}}$. Lower panel shows the activity of regulatory sensors in E12.5 embryos inserted at indicated positions. Expression pattern (WISH) of Kcnj2 and Sox 9 is shown for comparison. 


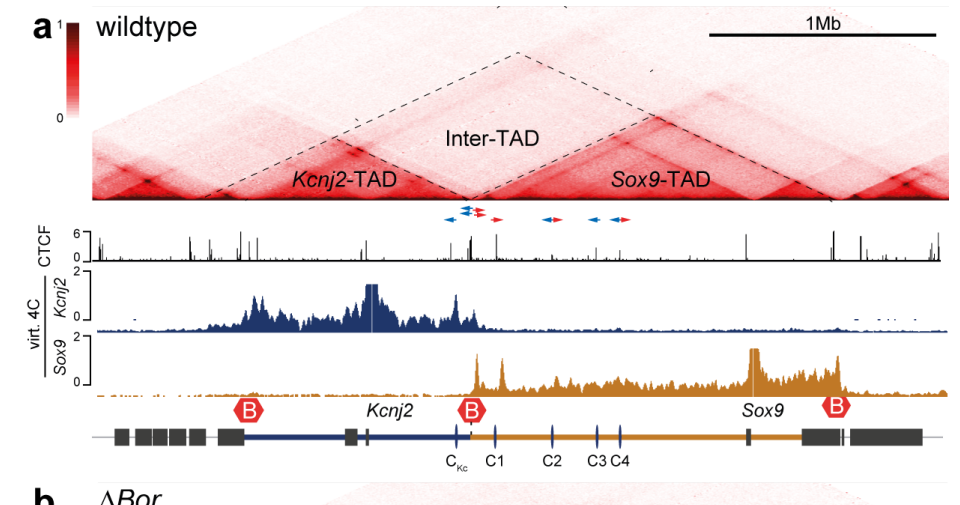

b $\Delta B o r$

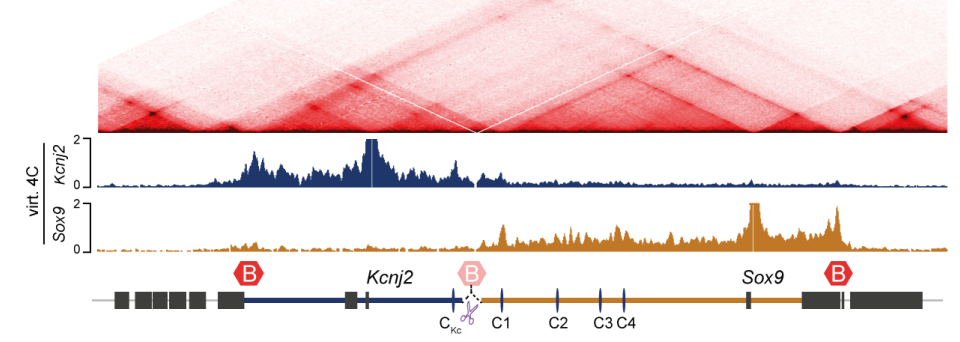

C

$\triangle B$ BorC1-4

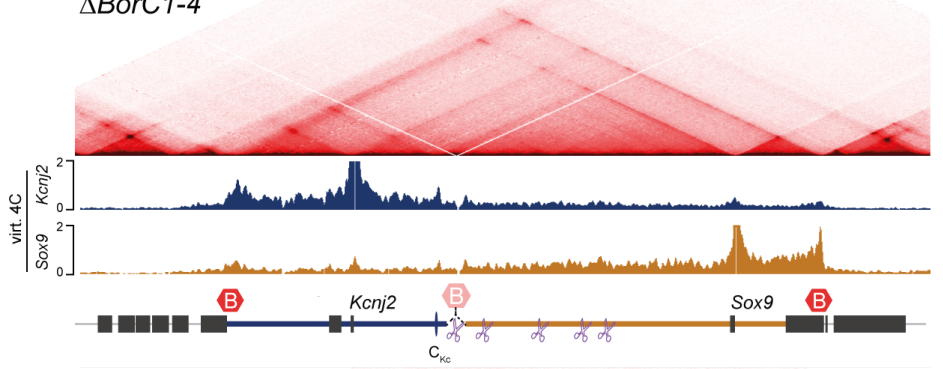

d $\triangle C T C F$

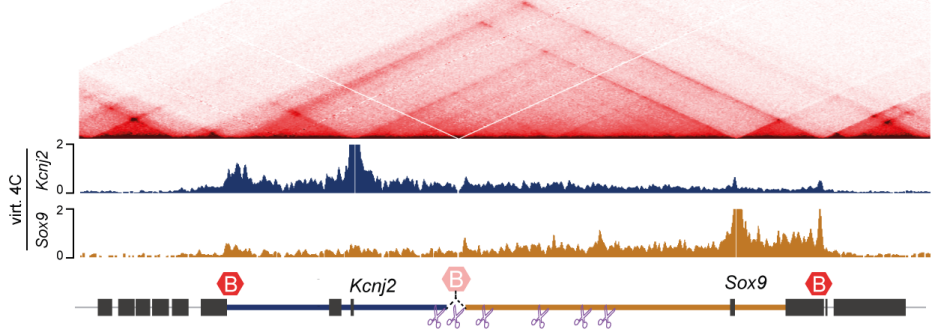

Figure 2 Progressive fusion of the Kcnj- and Sox9-TADs upon deletion of TAD boundary and intra-TAD CTCF sites.

cHiC from E12.5 mouse limb buds. Virtual 4C with viewpoints at the Kcnj2 (blue) or the Sox9 promoter (orange) below. CTCF ChIP-seq with binding site orientation (red/blue) at the boundary and intra-TAD are highlighted. Two-headed arrow indicates two oppositely oriented sites (FIMO $p<10^{-4}$ ) underlying the ChIP-seq peak. (A) Wildtype $\mathrm{cHiC}$. Dashed lines indicate Kcnj and Sox9-TADs and area of inter-TAD contacts. (B) 18kb-deletion ( $\triangle B$ Bor) of the TAD boundary leaves TAD configuration largely unchanged. (C) Deletion of the TAD boundary and targeted deletion of all four major intra-TAD CTCF-binding sites ( $\triangle B$ BorC1-C4) cause TAD fusion (D) $\triangle C T C F$ shows further TAD fusion upon deletion of the TAD boundary and all CTCF-binding-sites between the Kcnj2- and Sox9-promoter (C1-C4 and $\mathrm{C}_{\mathrm{KC}}$ ). 
a

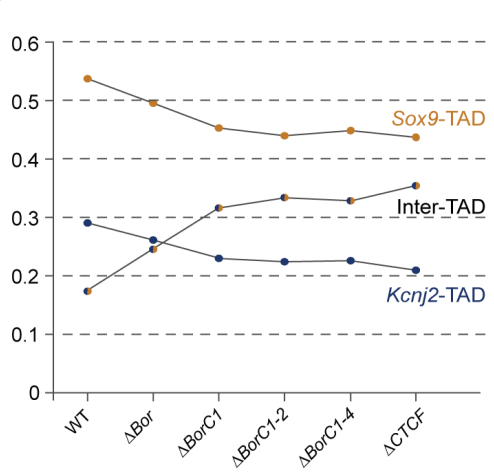

b

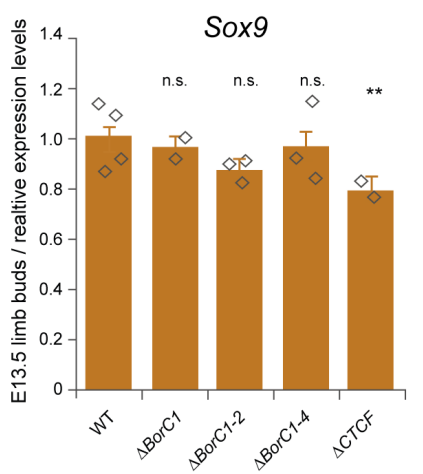

Kcnj2

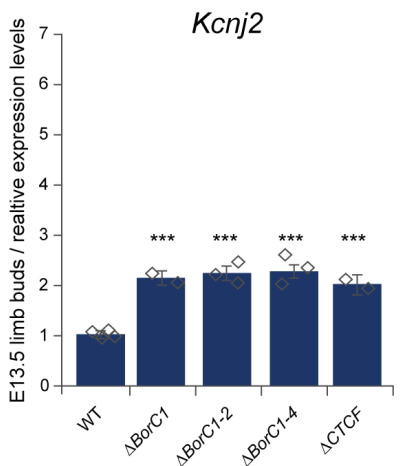

C
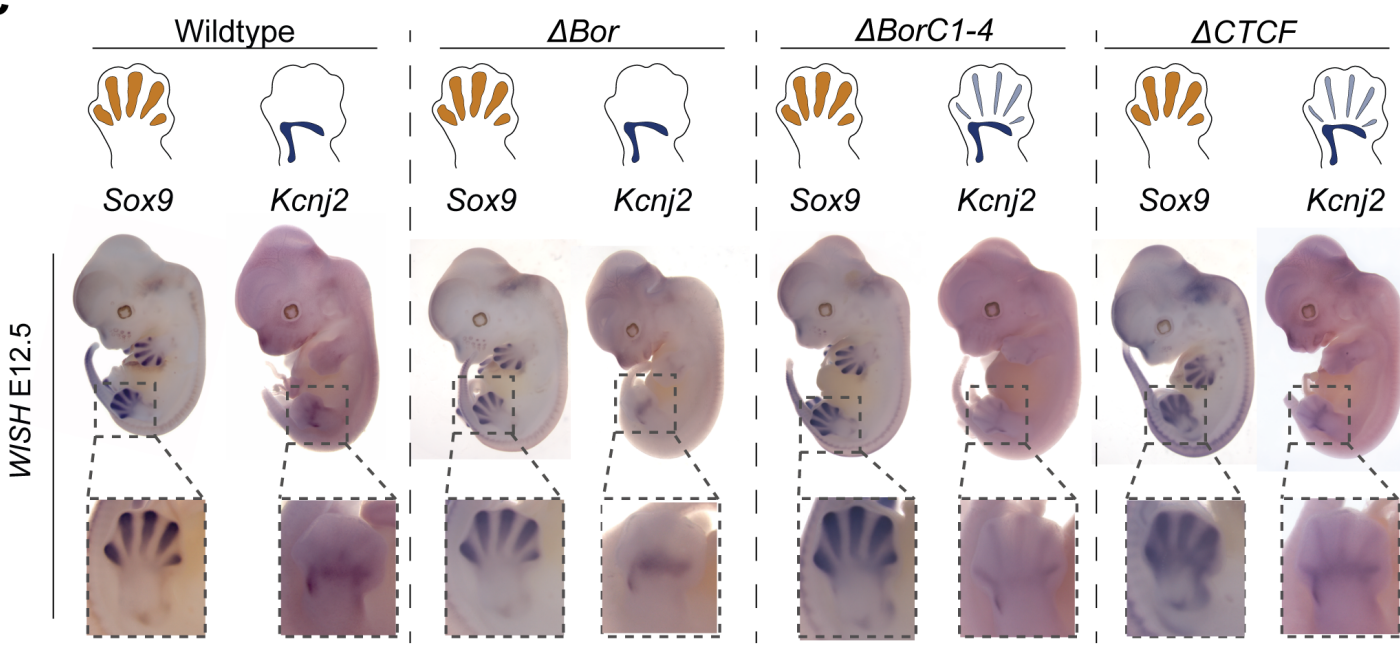

Figure 3: Effect of TAD fusion and deletion of CTCF binding sites on gene expression and phenotype.

(A) Change of interaction score induced by consecutive removal of CTCF sites. Percentage of contacts in the Kcnj2, and Sox9-TADs and between TADs (inter-TAD). The more CTCF-binding sites are deleted, the higher the contact frequency between the Kcnj2- and Sox9-TAD. (B) Relative gene expression of Kcnj2 and Sox9 in E13.5 limb buds measured by real-time qPCR. Values are normalized to Gapdh-expression (Wildtype $=1$ ). Bars represent the mean, error bars the standard deviation, diamonds indicate individual replicates. Significance in comparison to wildtype levels tested with unpaired t-test $\left(p<0.01:^{* *}, p<0.001:^{* * *}\right)$ (C) Expression pattern of Sox9 and Kcnj2 in E12.5 limbs (WISH). Schematic on top, whole-mount in situ hybridization below, detailed view of hindlimbs at the bottom. Sox9 is strongly expressed in the digit anlagen, whereas Kcnj2 is expressed weakly in the distal stylopod. Note no change in Sox 9 expression and low degree of digit expression of Kcnj2 only in $\triangle B$ BrC1-4 and $\triangle C T C F$. 

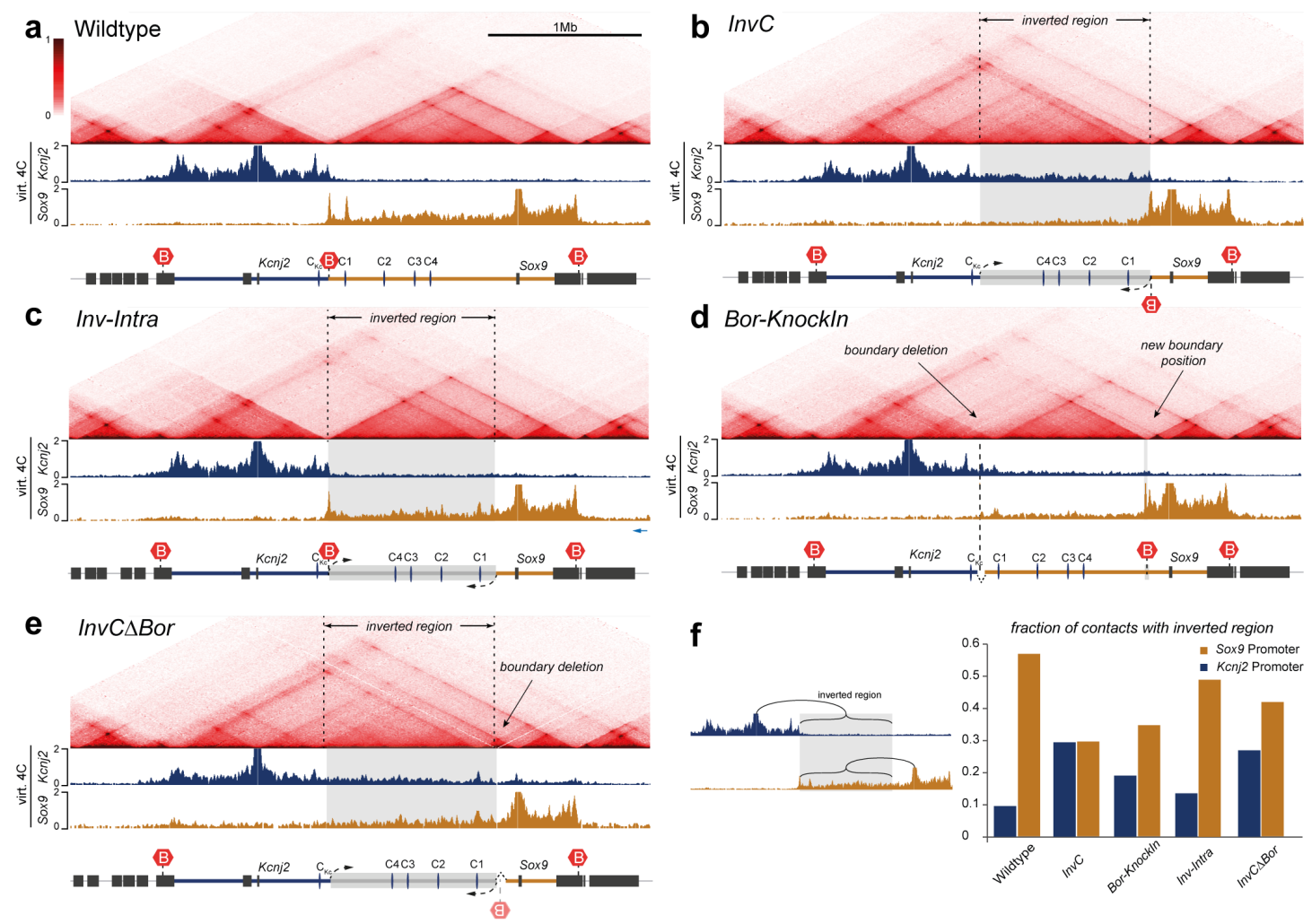

Figure 4: Boundaries and orientation of regulatory landscapes define TAD organization.

cHiC from E12.5 mouse limb buds, each mapped to a custom genome. 4C with viewpoint at the Kcnj2 (blue) or Sox9-promoter (orange) below. Grey box indicates the extent of inverted region. (A) Wildtype. (B) Inversion including boundary $(\operatorname{InvC})$ leads to fusion of the inverted region with the KCnj-TAD and separation of Sox9 from its regulatory domain. (C) Inversion excluding the boundary (Inv-Intra) has no major changes in TAD configuration. (D) Repositioning of boundary to a new position near Sox9 (Bor-Knockln) isolates of Sox9 from its TAD but does not cause the fusion with the Kcnj-TAD. (E) InvC inversion with deleted boundary (InvC $\triangle B o r$ ) causes fusion of both TADs and re-establishes Sox 9 contacts with its TAD. (F) Fraction of contacts with the inverted region for each allele. Bar diagram shows contacts of Kcnj2 (blue) or the Sox9 (orange) with the inverted region as a fraction of all contacts in the Kcnj and Sox9 TADs measured by $4 \mathrm{C}$. 
a

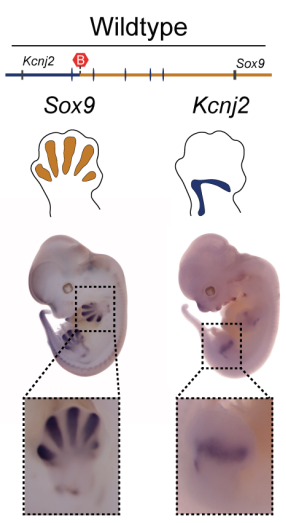

b

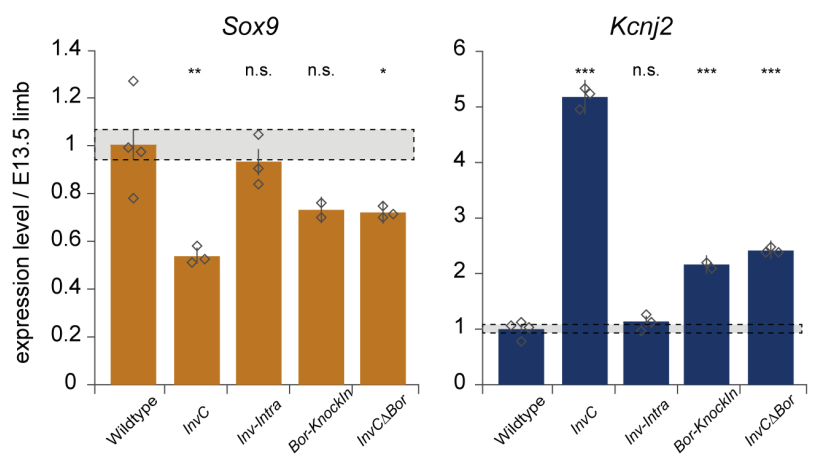

C
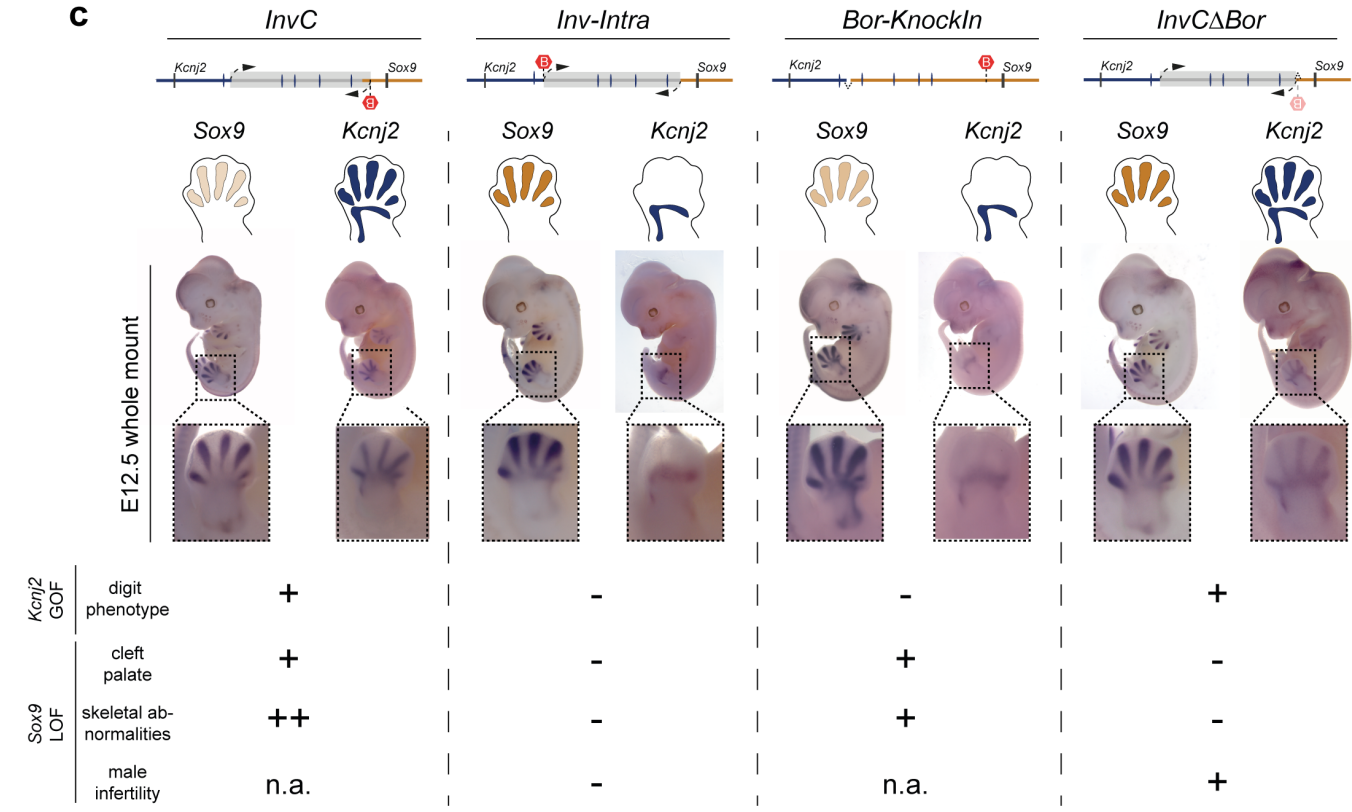
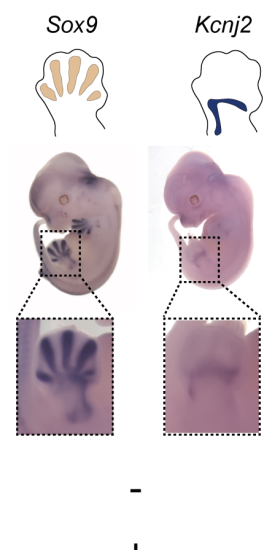

$+$

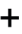

n.a.

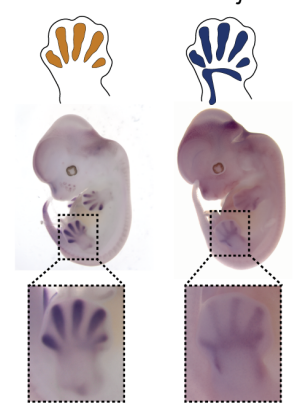

$+$

$-$

Figure 5: Re-direction of regulatory activity results in Kcnj2-misexpression and loss of Sox9 expression. (A) Wildtype gene expression pattern of Sox9 and Kcnj2 (B) Relative gene expression of Kcnj2 and Sox9 in E13.5 limb buds measured via real-time qPCR. Values are normalized to Gapdh-expression (Wildtype $=1$ ). Bars represent the mean, error bars the standard deviation, diamonds indicate individual replicates. Significance in comparison to wildtype levels tested with unpaired t-test (n.s. not significant, $p<0.05:^{*}, p<0.01$ : $^{* *}$, p<0.001:***) (C) Schematic of Structural Variant. Below, WISH of Sox9 and Kcnj2 in E12.5 limbs. Schematic on top, whole-mount in situ hybridization below, detailed view of hindlimbs at the bottom. Kcnj2 gains Sox9-like expression pattern in digits in InvC and InvC $\Delta B$ Bor. Sox 9 expression is visibly reduced in $\operatorname{InvC}$ embryos. Summary of phenotypes in animals induced by gain of $K c n j 2$ expression and/or loss of Sox 9 expression. 


\section{Methods}

\section{ES cell targeting and transgenic mouse strains}

ES cell culture was performed as described previously ${ }^{15}$. A list of sgRNAs used to generate the various deletions and inversions is given in Sup. Tab. XYZ. For targeting and re-targeting of CTCF-sites, sequence-verified ESC-lines were re-targeted with either one or two pX459sgRNAs. For each CTCF-site deletion, structural variants were excluded and modified CTCFsites for both alleles were verified through PCR-amplification of the cut-site, followed by sub-cloning and Sanger-sequencing of several PCR-products. Only if the successful modification of both alleles could be verified, the ESCs were used for further experiments. The results were later validated using the $\mathrm{cHi}-\mathrm{C}$ sequencing data.Embryos and live animals from ES cells were generated by di- or tetraploid complementation ${ }^{24}$. Genotyping was performed by PCR analysis.

\section{CRISPR-guided knock-in in mouse ESCs}

For targeting the Kcnj-Sox9-TAD boundary, a $6.3 \mathrm{~kb}$ construct containing the four CTCF sites (C1 site (mm9 chr11:111384818--111385832 followed by C2-4 chr11:111393908111399229) was cloned into a targeting vector with assymetric homology arms (HA1: chr11:112511756--112514691, , HA2: chr11:112514692-12519932) using standard cloning procedures.

For knock-in of targeting constructs without selection marker, the targeting construct was transfected in combination with a pX459-sgRNA vector. Importantly, the targeting construct did not contain either an intact PAM-site or guide-sequence. Puromycin selection and clonal ES cell line generation was performed as described previously. Successfully targeted ESClines were screened using PCR and validated for locus-specific integration after successful establishment of the ESC line. Validation of the homozygous TAD-boundary knock-in was performed bioinformatically using the $\mathrm{cHi}-\mathrm{C}$ data.

\section{LacZ-Sensor mouse lines}

The SB-Kcnj and SB-Sox9 alleles described in Franke et al. were used for remobilization of the $S B$ transgene, following the protocol in Ruf, et al. ${ }^{25}$, to generate new SB insertion sites (LacZ-Sensors) at the locus (Kcnj-TAD: SB20, SB16, SB24; Sox9-TAD: SB23, SB18). An additional LacZ-Sensors in the Sox9-TAD (mid-Sox9) was targeted directly using CRISPRguided knock-in as described above. Asymmetric homology arms ( 0.8 and $1.5 \mathrm{~kb})$ with mutated PAM sites and restriction sites for cloning of the LacZ-transgene were obtained from IDT, cloned into a plasmid vector. The beta-Globin-LacZ-transgene was then inserted into the Acc65I linearized targeting vector using Gibson assembly A list of primers used for 
cloning and genotyping targeting construct, and pX459-sgRNAs are provided in Supplementary Table 1.

\section{Generation of mice}

Mice from transgenic and genome edited ESCs were generated by di- or tetraploid aggregation (REF), maintained by crossing them with C57BI.6/J mice, and genotyped by PCR. Primers for genotyping can be provided upon request. All animal procedures were conducted as approved by the local authorities (LAGeSo Berlin) under the license number \#G0368/08 and \#G0247/13.

\section{Expression analysis}

RNA for qPCR was extracted from E13.5 mouse zeugopods. After dissection, samples were immediately frozen in liquid nitrogen, and individual embryos were genotyped. Tissue was lysed in RLT buffer and a .20 gauge syringe and RNA extraction was performed using the RNeasy Mini Kit (Qiagen) according to manufacturer's instruction. cDNA-synthesis was performed with SuperScriptIII RT (invitrogen) and polyT-Primers and qPCR was performed on an $\mathrm{ABI} 9700$.

\section{Whole-mount In Situ Hybridization}

E12.5 embryos were subjected to whole mount in situ hybridization using standard procedures. Sox9 and Kcnj2 probes were generated by PCR amplification using mouse limb bud cDNA (Supplementary Table 1).

\section{Micro-Computer Tomography}

Autopods of seven week old control and mutant mice were scanned using a Skyscan $1172 \mathrm{X}$ ray microtomography system (Brucker microCT, Belgium) at $5 \mu \mathrm{m}$ resolution. 3D model reconstruction was done with the Skyscan image analysis software CT-Analyser and CTvolume (Brucker microCT, Belgium).

\section{cHiC data processing}

Raw reads were preprocessed with cutadapt $\mathrm{v} 1.15^{26}$ to trim potential low quality bases (-q 20 -m 25) and potentially remaining sequencing adapters (-a and -A option with Illumina TruSeq adapter sequences according to the cutadapt documentation) at the 3' ends of the reads. Mapping, filtering, and deduplication of the short reads were performed with the HiCUP pipeline v0.5.10 ${ }^{27}$ (no size selection, Nofill: 1, Format: Sanger). The pipeline was set up with Bowtie2 v2.2.6 ${ }^{28}$ for mapping short reads to reference genome $\mathrm{mm} 9$. For inversions 
and the Border-KI allele, reads were also mapped to a customized genome, derived from $\mathrm{mm} 9$ based on the genotyping of the mutant ESC lines. Juicer tools $0.7 .5^{29}$ was used to generate binned contact maps from valid and unique read pairs with $M A P Q \geq 30$ and to normalize contact maps by Knight and Ruiz (KR) matrix balancing ${ }^{3,29,30}$ For the generation of contact maps, only reads pairs mapping to the enriched genomic region (chr11:109,010,001$114,878,000$ ) were considered and shifted by the offset of the enriched genomic region $(109,010,000 \mathrm{bp})$. For the import with Juicer tools, we used a custom chrom.sizes files containing only the size of the enriched part of the genome. Afterwards, KR normalized maps were exported at $10 \mathrm{~kb}$ resolution and coordinates were shifted back to their original values.

Subtraction maps were generated from KR normalized maps, which were normalized in a pair-wise manner before subtraction. To account for differences between two maps in their distance-dependent signal decay, maps were scaled jointly across their sub-diagonals. Therefore, the values of each sub-diagonal of one map were divided by the sum of this subdiagonal and multiplied by the average of these sums from both maps. Afterwards, the maps were scaled by $10^{6} /$ total sum. cHiC maps as well as subtractions maps were displayed as heatmaps in which (absolute) values above the $98.5^{\text {th }}$ percentile were truncated for visualization purposes.

\section{Virtual Capture-C}

In order to obtain individual interaction profiles for specific viewpoints with more finegrained binning, we created virtual Capture-C like interactions profiles from the same filtered bam files that were used for the $\mathrm{CHiC}$ maps. Paired-end reads with $\mathrm{MAPQ}>=30$ were considered in a profile when one mate mapped to the viewpoint region, while the other one mapped outside of it. Contacts of the viewpoint region with the rest of the genome were counted per restriction fragment. Afterwards, count data was binned to $1 \mathrm{~kb}$ bins. In case a restriction fragment overlapped with more than one bin, the counts were split proportionally. Afterwards, each profile was smoothed by averaging within a sliding window of $5 \mathrm{~kb}$ and scaled by $10^{3} / \mathrm{sum}$ of its counts within the enriched region. The viewpoint and a window $\pm 5 \mathrm{~kb}$ around it were excluded from the computation of the scaling factor. The profiles were generated with custom Java code using htsjdk v2.12.0 (https://samtools.github.io/htsjdk/).

\section{Interaction score between Kcnj2 TAD and Sox9 TAD}

The Kcnj2 TAD was manually defined as genomic region chr11:110,340,001-111,400,000 and the Sox9 TAD as chr11:111,400,001-113,030,000. Contact counts were summed within each 
TAD individually and within the region of the $\mathrm{cHiC}$ map containing the contacts between the two TADs. To avoid a strong influence of the main diagonal, only contacts spanning more than $100 \mathrm{~kb}$ were considered in this analysis. The three sums of contact counts were normalized to represent fractions adding up to 1 . Thus, the change of contact frequency between the two TADs was determined with respect to the intra-TAD contact frequency. For the calculation of the Sox9/Kcnj2 contacts with the centromeric Sox9 TAD, the virtual 4Cseq interaction data were further processed. The contacts with the "inverted region" represent the respective proportion of all Sox9/Kcnj2 promoter contacts in the Sox9 and Kcnj2 TADs. The contacts $<50 \mathrm{~kb}$ from the promoter were excluded from the calculation.

\section{Acknowledgements}

This study was supported by grants from the Deutsche Forschungsgemeinschaft to SM. We thank Judith Fiedler and Karol Macura (transgenic unit), Myriam Hochradel (sequencing core facility) of the MPIMG; Asita Stiege and Ute Fischer for help with cloning and cell culture and Norbert Brieske for in situ hybridizations. We also thank all members of the Mundlos lab for input and discussions.

\section{Author Contributions}

D.M.I. and S.M., conceived the project. A.D., D.M.I., and M.F. performed cHi-C, R.S. performed the computational analysis with input from M.V. and D.M.I.. D.M.I., A.D., S.A., and M.F. produced transgenics, carried out transgenic validation, and performed expression/phenotypic analysis. C.P. performed ATAC-seq, A.D., I.J. and D.M.I. ChIP-seq. B.T. oversaw sequencing of the $\mathrm{CHi}-\mathrm{C}, \mathrm{ChIP-}$, and ATAC-seq. L.W. performed morula aggregation. W-L.C. performed micro-CT analysis. D.M.I., A.D., and S.M. wrote the manuscript with input from the authors.

\section{Data availability}

Datasets are available through the Gene Expression Omnibus (GEO) under accession number GSE78109 and GSE125294. Secure token for reviewers: szudooiinjwfpej

\section{Author Information}

The authors declare no competing financial interests. Correspondence and requests for materials should be addressed to S.M. (mundlos@molgen.mpg.de) and D.M.I. (ibrahim@molgen.mpg.de) 


\section{References}

1. Bonev, B. \& Cavalli, G. Organization and function of the 3D genome. Nature Reviews Genetics 17,772 (2016).

2. Lieberman-Aiden, E. et al. Comprehensive mapping of long-range interactions reveals folding principles of the human genome. Science 326, 289-93 (2009).

3. Rao, S.S. et al. A 3D map of the human genome at kilobase resolution reveals principles of chromatin looping. Cell 159, 1665-80 (2014).

4. Nora, E.P. et al. Spatial partitioning of the regulatory landscape of the X-inactivation centre. Nature 485, 381-5 (2012).

5. Dixon, J.R. et al. Topological domains in mammalian genomes identified by analysis of chromatin interactions. Nature 485, 376-80 (2012).

6. Furlong, E.E.M. \& Levine, M. Developmental enhancers and chromosome topology. Science 361, 1341-1345 (2018).

7. Spielmann, M., Lupiáñez, D.G. \& Mundlos, S. Structural variation in the 3D genome. Nature Reviews Genetics 19, 453-467 (2018).

8. Nora, E.P. et al. Targeted Degradation of CTCF Decouples Local Insulation of Chromosome Domains from Genomic Compartmentalization. Cell 169, 930-944.e22 (2017).

9. Fudenberg, G. et al. Formation of Chromosomal Domains by Loop Extrusion. Cell Reports 15, 2038-2049 (2016).

10. Sanborn, A.L. et al. Chromatin extrusion explains key features of loop and domain formation in wild-type and engineered genomes. Proceedings of the National Academy of Sciences (2015).

11. Schwarzer, W. et al. Two independent modes of chromatin organization revealed by cohesin removal. Nature 551, 51 (2017).

12. Wutz, G. et al. Topologically associating domains and chromatin loops depend on cohesin and are regulated by CTCF, WAPL, and PDS5 proteins. The EMBO Journal 36, 3573-3599 (2017).

13. Busslinger, G.A. et al. Cohesin is positioned in mammalian genomes by transcription, CTCF and Wapl. Nature 544, 503 (2017).

14. Rao, S.S.P. et al. Cohesin Loss Eliminates All Loop Domains. Cell 171, 305-320.e24 (2017).

15. Franke, M. et al. Formation of new chromatin domains determines pathogenicity of genomic duplications. Nature 538, 265-269 (2016).

16. Lupianez, D.G. et al. Disruptions of topological chromatin domains cause pathogenic rewiring of gene-enhancer interactions. Cell 161, 1012-25 (2015).

17. Weischenfeldt, J. et al. Pan-cancer analysis of somatic copy-number alterations implicates IRS4 and IGF2 in enhancer hijacking. Nature Genetics 49, 65 (2016).

18. Bi, W., Deng, J.M., Zhang, Z., Behringer, R.R. \& de Crombrugghe, B. Sox9 is required for cartilage formation. Nature Genetics 22, 85 (1999).

19. Barrionuevo, F. et al. Homozygous Inactivation of Sox9 Causes Complete XY Sex Reversal in Mice1. Biology of Reproduction 74, 195-201 (2006).

20. Rodriguez-Carballo, E. et al. The HoxD cluster is a dynamic and resilient TAD boundary controlling the segregation of antagonistic regulatory landscapes. Genes Dev 31, 2264-2281 (2017).

21. Boija, A. et al. Transcription Factors Activate Genes through the Phase-Separation Capacity of Their Activation Domains. Cell 175, 1842-1855.e16 (2018).

22. Wendt, K.S. et al. Cohesin mediates transcriptional insulation by CCCTC-binding factor. Nature 451, 796 (2008).

23. Zabidi, M.A. et al. Enhancer-core-promoter specificity separates developmental and housekeeping gene regulation. Nature 518, 556 (2014).

24. Kraft, K. et al. Deletions, Inversions, Duplications: Engineering of Structural Variants using CRISPR/Cas in Mice. Cell Rep 10, 833-839 (2015).

25. Artus, J. \& Hadjantonakis, A.-K. Generation of Chimeras by Aggregation of Embryonic Stem Cells with Diploid or Tetraploid Mouse Embryos. in Transgenic Mouse Methods and Protocols, Vol. 693 (eds. Hofker, M.H. \& van Deursen, J.) 37-56 (Humana Press, 2011).

26. Martin, M. Cutadapt removes adapter sequences from high-throughput sequencing reads. 2011 17, 3 (2011).

27. Wingett, S. et al. HiCUP: pipeline for mapping and processing Hi-C data. in F1000Research Vol. 41310 (2015). 
bioRxiv preprint doi: https://doi.org/10.1101/566562; this version posted March 5, 2019. The copyright holder for this preprint (which was

not certified by peer review) is the author/funder, who has granted bioRxiv a license to display the preprint in perpetuity. It is made available under aCC-BY-NC-ND 4.0 International license.

28. Langmead, B. \& Salzberg, S.L. Fast gapped-read alignment with Bowtie 2. Nat Methods 9, 357-9 (2012).

29. Durand, N.C. et al. Juicer Provides a One-Click System for Analyzing Loop-Resolution Hi-C Experiments. Cell Systems 3, 95-98 (2016).

30. Knight, P.A. \& Ruiz, D. A fast algorithm for matrix balancing. IMA Journal of Numerical Analysis 33, 1029-1047 (2013). 


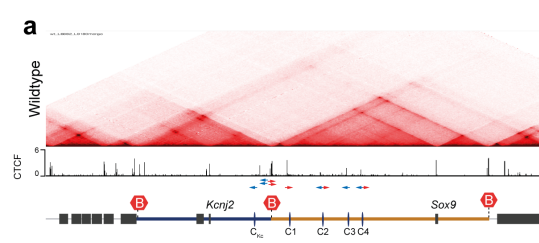

b
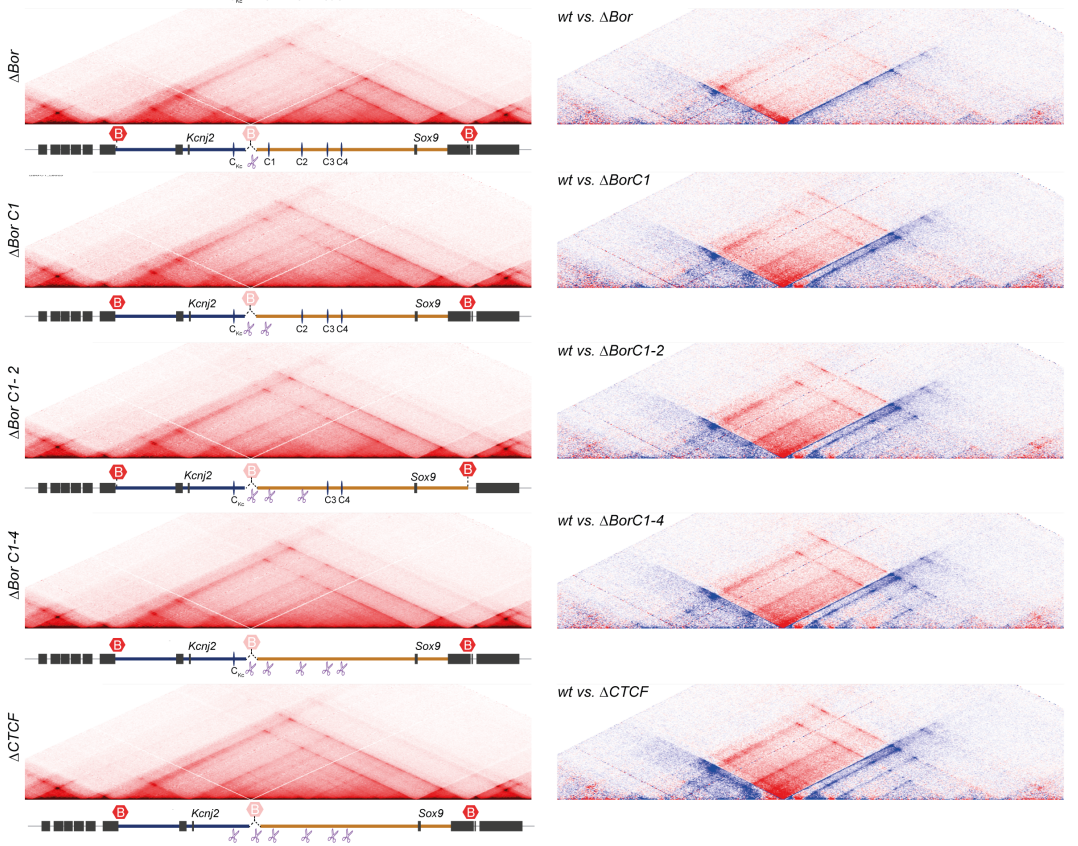

Figure S1: Gradual fusion upon progressive CTCF site deletion.

(A) CHiC chromatin interactions from wildtype and mutant E12.5 mouse embryonic limb buds. CTCF ChIP-seq with binding site orientation (red/blue) at the boundary and intra-TAD are highlighted. Two-headed arrow indicates two significant motifs (FIMO, $p<10^{-4}$ ) underlying the ChIP-seq peak. Virtual 4C from Kcnj2 (blue) and Sox9 (orange) below each map. Progressive deletion of CTCF sites causes progressively increasing interaction between Sox9- and Kcnj-TADs (B) Subtraction maps of wildtype vs. mutant for the same region show increasingly more (red) interactions in the mutants and loss (blue) of the intra-TAD CTCFmediated contacts. 

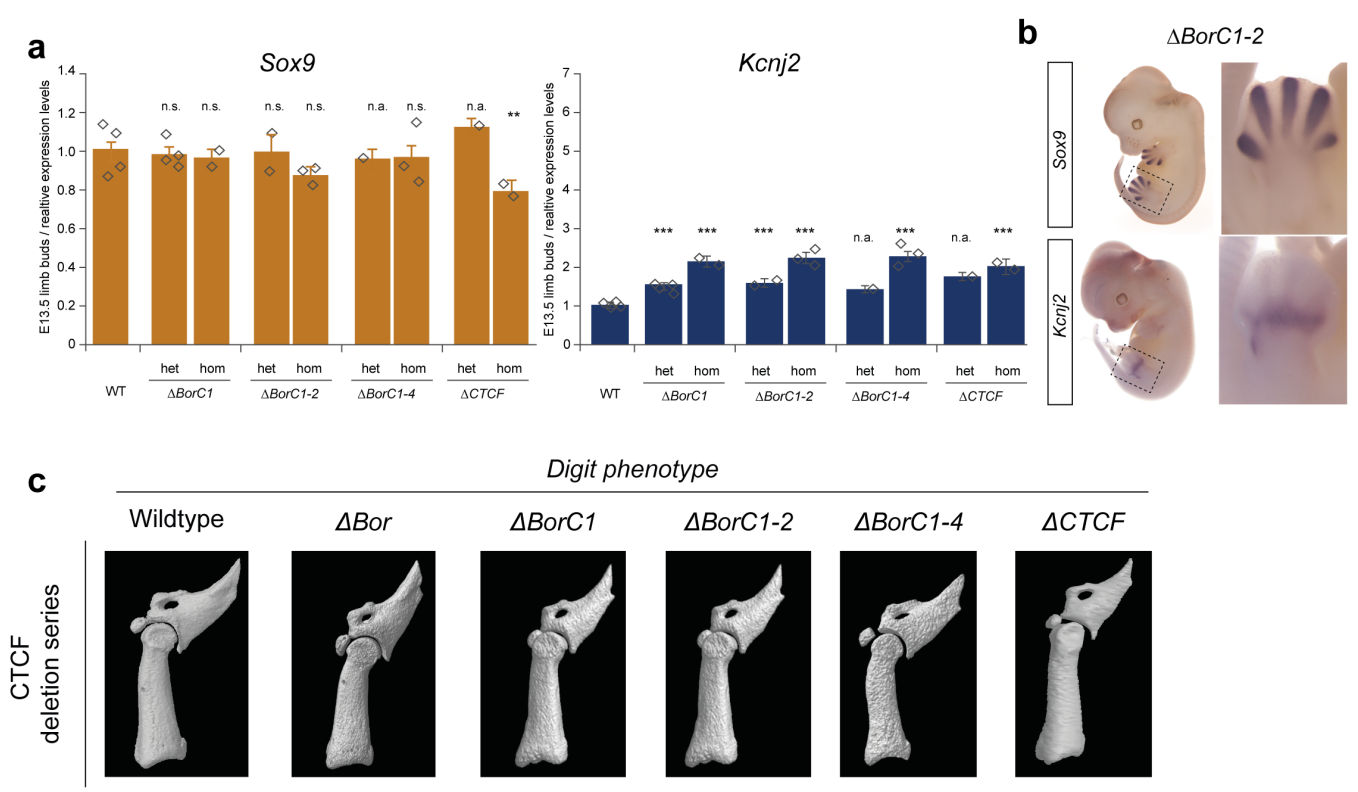

Figure S2

Figure S2: Minor gene expression changes and absence of digit phenotypes upon CTCF-site deletion.

(A) Gapdh normalized expression of Kcnj2 and Sox9 in E13.5 limb buds from heterozygous and homozygous littermates measured via qRT-PCR (wildtype $=1$ ). Bars represent the mean, error bars the standard deviation. Significance relative to wildtype levels tested via unpaired t-test (n.s.: not significant, $p<0.05:^{*}, p<0.01:^{* *}, p<0.001:^{* * *}$, n.a.: not available) (B) Normal Kcnj2 and Sox9 expression pattern in $\triangle$ BorC1-2 E12.5 embryos by WISH. Zoom-In shows hindlimb in detail (C) 3D micro-computed tomography scan of terminal phalanges from wildtype and mutant adults (7-12w). 
WT
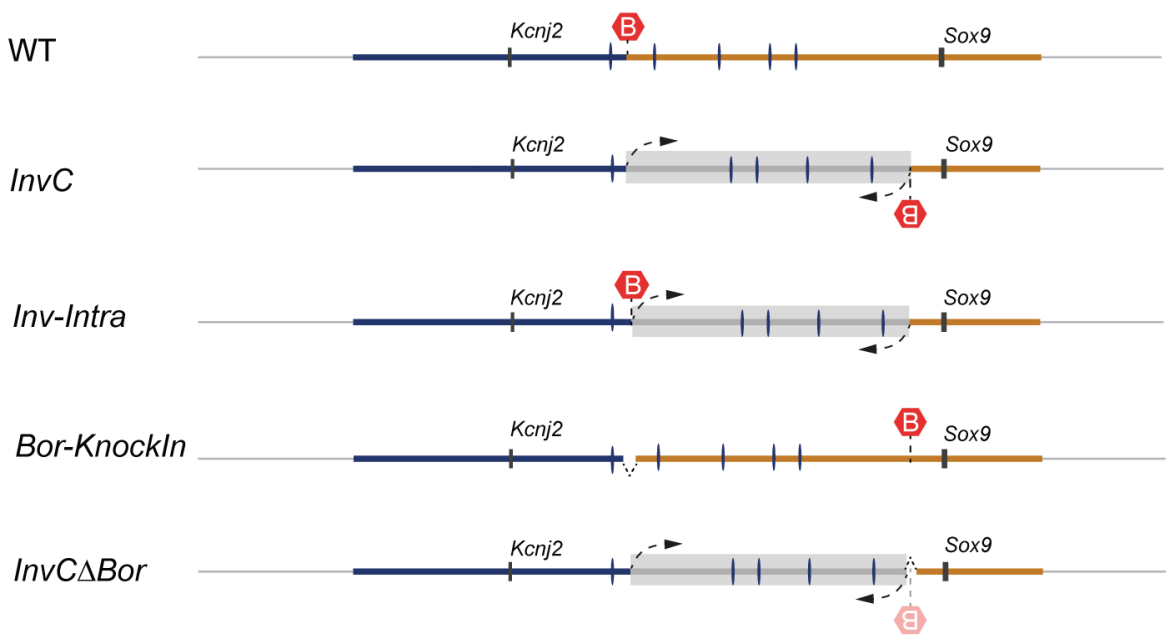

Figure S3

Figure S3: Schematic of the inversion series at the Kcnj-Sox9 locus

Position of the Kcnj2 and Sox9 genes, TAD boundary (red hexagons), and CTCF sites (blue ticks) in wildtype and mutant genomes are indicated. Grey boxes indicate the extent of the inverted region. 


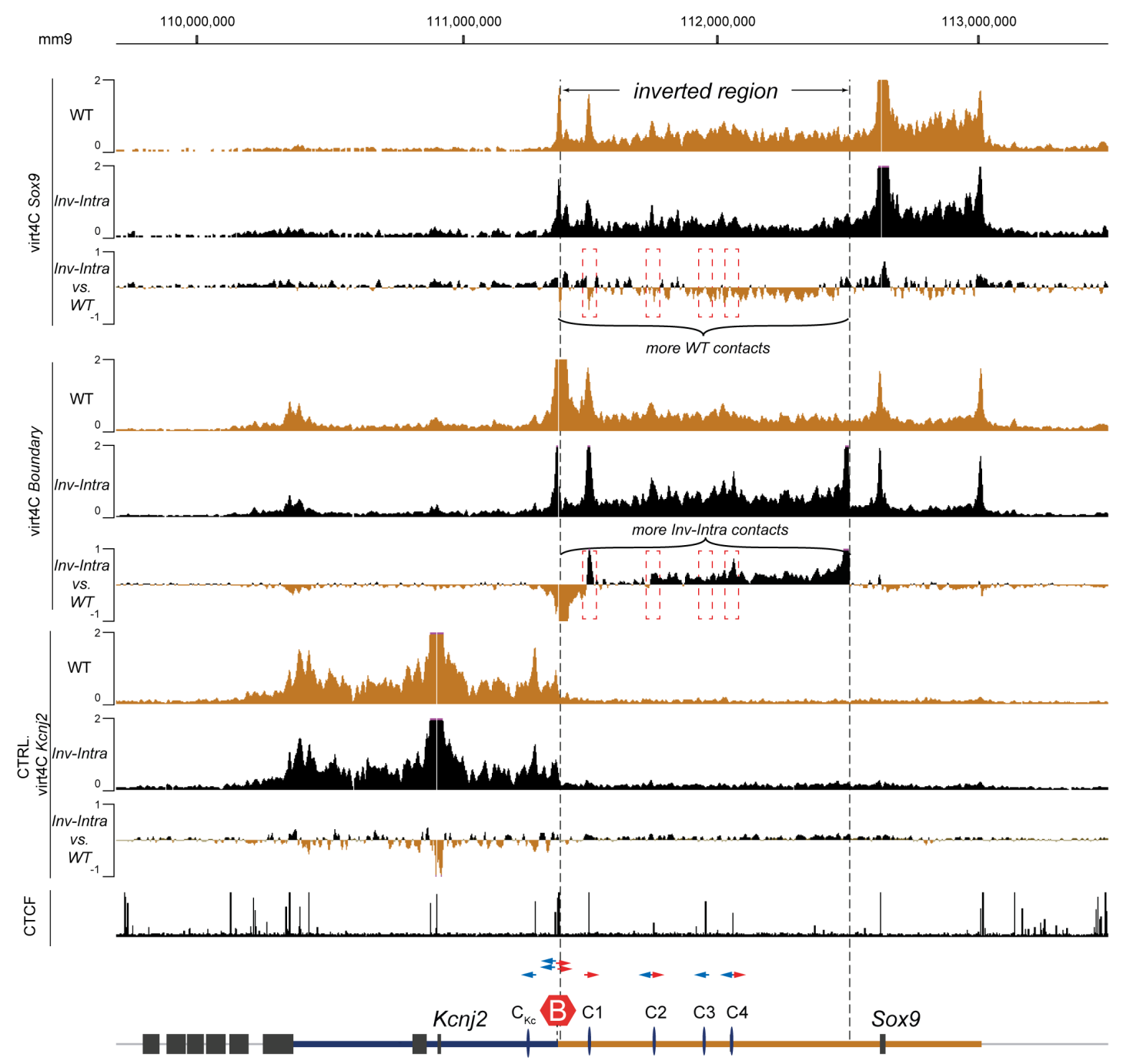

Figure S4

Figure S4: Subtle differences in interactions upon intra-TAD inversion (Inv-Intra).

Virtual 4C-profiles derived from cHi-C maps (wildtype and Inv-Intra) mapped to a wildtype genome. 4C-profiles from viewpoints at Sox9, the TAD boundary, and Kcnj2 are shown for wildtype (orange) and Inv-Intra (orange). Subtraction of the two profiles shows systematically more contacts at the Boundary and Sox9 viewpoints depending on orientation of TAD substructure for the. The control viewpoint (Kcnj2) shows no systematic changes. Note changes in interaction frequency of Sox 9 or the TAD boundary upon inversion of the TAD substructure. Dashed boxes indicate changes in interaction at the C1-C4 CTCF sites. 
a

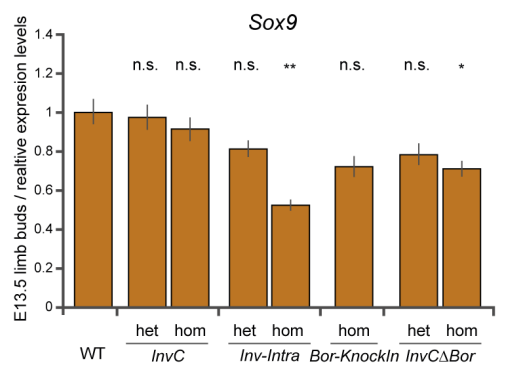

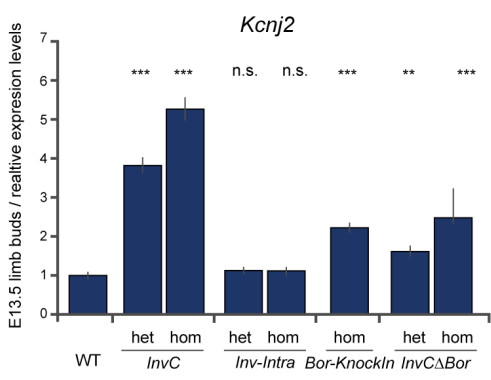

Inv-Intra

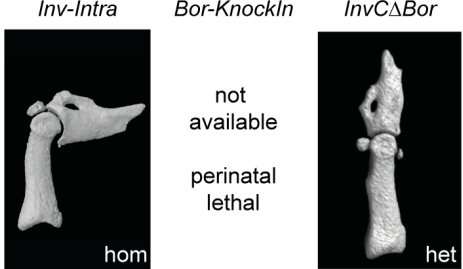

d

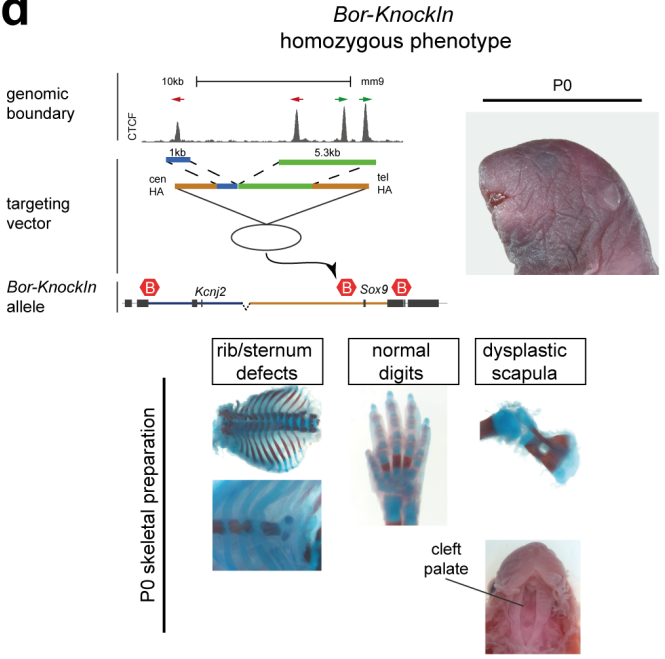

Figure S5

Figure S5: Re-direction of genomic interactions leads to Sox9 loss and Kcnj2 gain in expression and developmental phenotypes.

(A) Gapdh normalized expression of Kcnj2 and Sox9 in E13.5 limb buds from heterozygous and homozygous littermates measured via qRT-PCR (wildtype $=1$ ). Error bars represent the std, deviation the mean, diamonds the individual data points. Significance relative to wildtype (unpaired t-test; n.s.: not significant, $p<0.05:^{*}, p<0.01:^{* *}, p<0.001:^{* * *}$ ). Note the allele-specific regulatory changes in InvC and InvCABor littermates. Homozygous Bor-KnockIn embryos were generated by tetraploid aggregation. (B) Loss of dorsal flexion, sesamoid bones, and claw-shaped form of the terminal phalanx in adult (7-12w) InvC and InvCABor animals shown by 3D micro-computed tomography. (C) Homozygous InvC phenotype in E18.5 littermates. Top, lateral view of the head, note short snout and micrognathy in the homozygous embryo. Bottom, Skeletal preparation of littermates show Sox9-LOF characteristic skeletal defects (sternum/rib defects, delayed ossification, dysplastic digits and scapula, cleft palate). (D) Homozygous Bor-Knockln phenotype in PO-animals derived from tetraploid aggregation. Top, schematic of the targeting construct containing the four CTCF sites of the TAD boundary (6.3 kb). Homology arms are 2 and $4 \mathrm{~kb}$. Top right: lateral view of PO newborn, note short snout and micrognathy. Bottom: Bor-KnockIn phenotype displays Sox9-LOF like defects. Note the overall milder defects compared to InvC and absence of a digit phenotype. 